\title{
Reductions in Myeloid-Derived Suppressor Cells and Lung Metastases using AZD4547 Treatment of a Metastatic Murine Breast Tumor Model
}

\author{
Li Liu, ${ }^{a, d}$ Ting Hong Ye $\mathrm{e}^{\mathrm{b}, \mathrm{d}}$ Yuan Ping Han ${ }^{\mathrm{c}}$ Hang Song ${ }^{\mathrm{a}}$ Yong Kui Zhang ${ }^{\mathrm{a}}$ \\ Yong Xia ${ }^{b} \quad$ Ning Yu Wang ${ }^{b}$ Ying Xiong $^{b}$ Xue Jiao Song ${ }^{b}$ Yong Xia Zhu ${ }^{b}$ \\ De Liang Lib Jun Zeng ${ }^{b}$ Kai Ran ${ }^{b}$ Cui Ting Penga,b Yu Quan Weib Luo Ting Yua,b \\ aDepartment of Pharmaceutical and Bioengineering, School of Chemical Engineering, Sichuan \\ University, ${ }^{b}$ State Key Laboratory of Biotherapy and Cancer Center, West China Hospital, West China \\ Medical School, Sichuan University, 'School of Life Sciences, Sichuan University, Chengdu, China; \\ 'These authors contributed equally to this work
}

\section{Key Words}

Breast cancer • FGFR inhibitor • AZD4547 • Metastasis • MDSCs • T cells

\begin{abstract}
Background: AZD4547, a small-molecule inhibitor targeting the tyrosine kinase of Fibroblast Growth Factor Receptors (FGFRs), is currently under phase II clinical study for human subjects having breast cancer, while the underlying mechanism remains elusive. The aim of this study is to explore the potential mechanism by which AZD4547 inhibits breast tumor lung metastases at the level of the tumor microenvironment. Methods: First, through in vitro experiments, we investigated the efficacy of the FGFRs inhibitor AZD4547 on 4T1 tumor cells for their proliferation, apoptosis, migration, and invasion. Second, by in vivo animal experiments, we evaluated the effects of AZD4547 on tumor growth and lung metastases in 4T1 tumor-bearing mice. Finally, we examined the impact of AZD4547 on the infiltration of myeloid-derived suppressor cells (MDSCs) in lung, spleens, peripheral blood and tumor. Results: Through this study we found that AZD4547 could efficiently suppress tumor 4T1 cells through restraining their proliferation, blocking migration and invasion, and inducing apoptosis in vitro. In animal model we also demonstrated that AZD4547 was able to inhibit tumor growth and lung metastases, consistent with the decreased MDSCs accumulation in the tumor and lung tissues, respectively. Moreover, the reduced number of MDSCs in peripheral blood and spleens were also observed in the AZD4547-treated mice. Importantly, through the AZD4547 treatment, the $\mathrm{CD}^{+}$and $\mathrm{CD}^{+}{ }^{+} \mathrm{T}$-cells were significantly increased in tumor and spleens. Conclusion: Our studies showed that AZD4547 can inhibit breast cancer cell proliferation, induce its apoptosis and block migration and invasion in vitro and suppress tumor growth and lung metastases by modulating the tumor immunologic microenvironment in vivo.

Copyright $\odot 2014$ S. Karger AG, Basel




\section{Introduction}

A recent epidemiological study shows that breast cancer is expected to account for about $29 \%$ of all cancers among women in 2013 [1]. And its poor prognosis is always related to invasion and metastasis, which are typical features for breast cancer patients in their advanced tumor progression [2,3]. In a typical metastasis process, tumor cells have to disseminate and intravasate into blood vessels at the primary tumor site, travel through the vascular systems, and then extravasate out from blood vessel to colonialize in the parenchyma of distal tissues. In the distant organ, they may escape from host immune surveillance to survive and grow [4]. Therefore, targeting any of these steps could be served as potential treatment for metastatic tumor including breast cancer.

On the other hand, the tumor microenvironment is now recognized as an important participant in tumor progression, particularly as it relates to invasive and metastatic progression. Consequently, targeting the tumor microenvironment has been the focus study for developing new anti-cancer therapies in recent years [5]. Myeloid-derived suppressor cells (MDSCs) expanded in pathological conditions are a heterogeneous population of activated immature myeloid cells (IMCs), and unable to fully differentiate into mature cells in such forms as macrophages, granulocytes, and dendritic cells [6]. The expansion of MDSCs is often associated with malignant tumor growth. These cells have been attributed to the regulation of angiogenesis, promotion of metastasis and induction of immune suppression $[7,8]$. Importantly, the increased levels of circulating MDSCs are correlated extensively with metastatic tumor burden in patients with breast cancer [9]. MDSCs are usually defined as $\mathrm{Gr}-1^{+} / \mathrm{CD} 11 \mathrm{~b}^{+}$cells in mice, which are unable to process and present antigens, and therefore are unable to induce tumor specific $\mathrm{CD} 4^{+} \mathrm{T}$-cells, $\mathrm{CD}^{+}{ }^{+} \mathrm{T}$-cells in $\mathrm{T}$ cell mediated immune responses against the tumor $[10,11]$. Taken together, it is assumed that the elimination of these cells could significantly improve anti-tumor responses and enhance cancer immunotherapy efficacy.

Fibroblast growth factor receptors (FGFRs) belong to the receptor tyrosine kinase superfamily. Activation of FGFRs, through interaction with FGF ligands; plays several crucial roles in multiple biologic processes including tumor cells apoptosis, angiogenesis and migration. Deregulation of FGFR signaling has been reported in clinical samples of breast [12], multiple myeloma [13], endometrial [14], gastric [15], and prostate cancers [16]. AZD4547 is an orally bioavailable, potent and highly selective ATP-competitive smallmolecule inhibitor against FGFRs tyrosine kinase. Recently, it was reported that AZD4547 selectively inhibited FGFR1 amplification in non-small cell lung cancer [17] as well as FGFR2 amplification in gastric cancer [18]. In addition, AZD4547 is under investigation in breast cancer at Phase II clinical trial.

In this study, we showed that AZD4547 could inhibit breast cancer proliferation and metastasis, and induce its apoptosis in vitro. Importantly, we also found that AZD4547 suppressed 4T1 tumor growth and reduced MDSCs accumulation in lung, tumor, spleens and peripheral blood in vivo. In particular, we found that AZD4547could inhibited tumorinduced MDSCs accumulation while conversely elevated T-cells to suppress tumor metastasis and growth. Thus, our study uncovered a potential new mechanism for AZD4547 on its therapeutic application.

\section{Materials and Methods}

AZD4547

$\mathrm{N}$-[5-[2-(3, 5-Dimethoxyphenyl)ethyl]-2H-pyrazol-3-yl]-4-(3, 5-diemthylpiperazin-1-yl) benzamide (AZD4547) was purchased from Selleck Chemicals (USA). For in vitro studies, AZD4547 was dissolved in dimethyl sulfoxide (DMSO) at a stock concentration of $20 \mathrm{mM}$ and stored at $-20{ }^{\circ} \mathrm{C}$. The working dosage was freshly diluted in the relevant medium to a final DMSO concentration of less than $0.1 \%$. For in vivo studies, mice were given AZD4547 (5 mg/kg) or vehicle control every other day by intraperitoneal injection. 
Liu et al.: AZD4547 Inhibits Metastasis in Breast Tumor Model

\section{Materials}

3-(4, 5)-dimethylthiahiazo (-z-y1) -2, 5 -di-phenytetrazolium bromide (MTT) and DMSO were purchased from Sigma Chemical Co. (St. Louis, MO). Annexin V-FITC apoptosis detection kit was purchased from Roche (Indianapolis, IN). The antibodies against $\beta$-actin and cleaved-caspase- 3 were purchased from Cell Signaling Technology Company (Beverly, MA). Anti-CD11b, anti-Gr1, anti-CD4, anti-CD8 and transwell plate were obtained from BD PharMingen. Mtrigel were purchased from BD Bioscience (San Jose, Amersham, UK).

\section{Cell culture}

4T1 (mouse breast tumor cell line), MDA-MB-231, MDA-MB-468 (human breast tumor cell lines), CT26 (mouse colorectal carcinoma cell line) and SW620, SW480, HCT116 (human colorectal carcinoma cell lines) were purchased from the American Type Culture Collection (ATCC, Rockville, MD, USA). Cells were cultured in RPMI-1640 or Dulbecco's Modified Eagle's Medium (DMEM) media supplemented with $10 \%$ fetal bovine serum (FBS; Gibco, Auckland, N.Z) and $0.1 \%$ antibiotic-antimycotic solution. Cells were maintained in a 37 ${ }^{\circ} \mathrm{C}$ incubator with $5 \%$ humidified $\mathrm{CO}_{2}$ atmosphere.

\section{Cell proliferation assay}

The cell viability of AZD4547 treated cell was performed by MTT assay. Briefly, the exponentially growing cells $2-4 \times 10^{3}$ cells in $100 \mu \mathrm{L}$ mediums were seeded in 96 -well plates for 24 hours. Then various concentrations of AZD4547 in $100 \mu \mathrm{L}$ mediums were added to each well and incubate for 24, 48, 72 hours respectively. A volume of $20 \mu \mathrm{L}$ of MTT solution $(5 \mathrm{mg} / \mathrm{ml})$ was added to each well and incubated for additional 2-4 hours. Finally the medium was discarded and $150 \mu \mathrm{L}$ of DMSO was added to each well for 15-20 minutes. The absorbance of each well was measured at $570 \mathrm{~nm}$ wavelength with Spectra MAXM5 microplate spectrophotometer (Molecular Devices) and the percentage of inhibition was calculated. The results were obtained from at least three separate experiments.

\section{Colony formation assay}

To test the survival of 4T1 treated with AZD4547, the 4T1 cells (200-300 cells/well) were seeded in 6-well plates and incubated for $24 \mathrm{~h}$ at $37{ }^{\circ} \mathrm{C}$, followed by various concentrations of AZD4547 treatment (0-2 $\mu \mathrm{M})$ for ten days with fresh medium. After washing with phosphate-buffered saline (PBS), colonies were fixed with $4 \%$ paraformaldehyde and stained with $0.5 \%$ crystal violet. Colonies with > 50 cells were counted under microscope.

\section{Transwell invasion and migration assays}

Invasion assay was conducted as previously described with some modifications [19]. Briefly, matrigel diluted $1: 2$ in serum-free medium was coated to the top chamber of 24 -well transwell plate $(8 \mu \mathrm{m}$ pore size) for $90 \mathrm{~min}$ at $37^{\circ} \mathrm{C}$. After matrigel polymerization, the bottom chambers were filled with $600 \mu \mathrm{L}$ RPMI1640 medium with $10 \%$ FBS and the top chambers were seeded with $1 \times 10^{5} 4 \mathrm{~T} 1$ cells in $100 \mu \mathrm{L}$ RPMI-1640 serum-free medium. Various concentrations of AZD4547 (0-10 $\mu \mathrm{M})$ were added to top chambers and the plate were incubated for $24 \mathrm{~h}$. The invasion was stopped by scraping non-migrated cells on the top chambers with a cotton swab and washed with PBS. Invaded cells were fixed with methanol and stained with $0.5 \%$ Crystal Violet. Migration assay was performed according to previous studies with some modifications [20]. Briefly, the top chambers were seeded with $1 \times 10^{5} 4 \mathrm{~T} 1$ cells in $100 \mu \mathrm{L}$ RPMI-1640 serum-free medium, and $600 \mu \mathrm{L}$ RPMI-1640 medium with $10 \%$ FBS was added at the bottom. Various concentrations of AZD4547 (0$10 \mu \mathrm{M}$ ) were added in both chambers and the plate were incubated for $24 \mathrm{~h}$. Non-migrated cells on the top chambers were removed using a cotton swab and washed with PBS. Migrated cells were fixed with methanol and stained with $0.5 \%$ Crystal Violet. Images were taken using a ZEISS digital microscope and with invading cells being counted by manual counting. Transwell invasion and migration assays were performed three times.

\section{Cell apoptosis analysis by Flow Cytometry (FCM)}

To further confirm the apoptosis inducing effect of AZD4547, the cell apoptosis was analyzed as described previously with slight modifications [21]. Briefly, 4T1 cells $\left(1 \times 10^{5}\right)$ were seeded in 6 -well plates. Then the cells were treated with various concentrations of AZD4547 (0-20 $\mu \mathrm{M})$ the next day for 24 hours 
Liu et al.: AZD4547 Inhibits Metastasis in Breast Tumor Model

as described above. Both attached cells and floating cells were harvested and washed with cold PBS twice. Cells were stained with Annexin V-FITC and propidium iodide (PI) according to the supplier's instructions followed by detecting with FCM. Finally, the data were analyzed by Flow Jo software.

\section{Western blot analysis}

Western blot analysis was performed as described previously [22]. Cells were washed with cold PBS twice and lysed in RIPA buffer after treatment with AZD 4547 for $48 \mathrm{~h}$ and the lysates were centrifuged at $13,000 \mathrm{~g}$ for $15 \mathrm{~min}$ at $4{ }^{\circ} \mathrm{C}$. The supernatant was harvested and the protein concentration was measured by the Lowry method. Equal amounts of total proteins were subjected to sodium dodecyl sulfate-polyacrylamide gel electrophoresis (SDS-PAGE) and transferred onto polyvinylidene fluoride (PVDF) membranes. After electrophoresis, the membranes were blocked for $1 \mathrm{~h}$ at room temperature and incubated overnight at 4 ${ }^{\circ} \mathrm{C}$ with the respective primary antibodies followed by the secondary antibody conjugated to horseradish peroxidase. The immunostaining signal was identified using the enhanced chemiluminescence system (Amersham, Piscataway, NJ).

\section{Animal models and drug administration}

All mouse protocols were approved by the Animal Care and Use Committee of Sichuan University (Chengdu, Sichuan, China). For subcutaneous tumor model, $100 \mu \mathrm{L}$ serum-free medium containing $1 \times 10^{6}$ $4 \mathrm{~T} 1$ cells were injected subcutaneously into the right flank of the seven-week-old female BALB/c mice. After one week, when average tumor volume reached approximately 60 to $80 \mathrm{~mm}^{3}$, mice were randomly divided into 2 groups and given AZD4547 at the dose of $5 \mathrm{mg} / \mathrm{kg}$ or vehicle control every other day by intraperitoneal injection. Tumor size and body weight were measured every other day during the treatment. The tumor volume was calculated according to the following formula by caliper: volume $\left(\mathrm{mm}^{3}\right)=0.5 \times$ length $(\mathrm{mm}) \times$ width $\left(\mathrm{mm}^{2}\right)$.

\section{Analysis tissues by FCM and single-cell sorting}

We prepared single-cell suspensions by mechanic dispersion of lung, tumor, spleens or peripheral blood tissues [23]. $1 \times 10^{6}$ freshly single-cell suspensions were prepared in PBS and were labeled with fluorescence-conjugated antibodies. We collected fluorescence data on FACSCalibur flow cytometer (BD Biosciences) and analyzed them using Flow Jo software.

\section{Statistical analysis}

Data were expressed as mean \pm SD from three independent experiments. The 2-tailed Student's test was employed to assess the statistical significance of difference between control groups and AZD4547treated groups. In all statistical analysis, ${ }^{*}, \mathrm{p}<0.05 ;{ }^{* *}, \mathrm{p}<0.01 ;^{* *}, \mathrm{p}<0.001$;

\section{Results}

AZD4547 inhibits tumor cell growth in vitro

In order to investigate the tumor cell growth inhibitory properties of AZD4547, we treated a panel of seven established cancer cell lines with AZD4547 for 24, 48, 72 hours respectively, and then assayed cell viability by MTT assay. In 4T1, MDA-MB-468 and HCT116 cell lines, the $\mathrm{IC}_{50}$ of AZD4547 were calculated as $0.64 \mu \mathrm{M}, 4.9 \mu \mathrm{M}$ and $15.9 \mu \mathrm{M}$, respectively, and displayed relatively weak activities against other cancer cell lines (Table 1). These results showed that among the tested tumor cell lines, 4T1 was the most sensitive one to AZD4547. For such regard, we chose this cell line for further experiments. Importantly, these results suggested that AZD4547 could selectively inhibit $4 \mathrm{~T} 1$ cell proliferation in a concentrationand time-dependent manner (Fig. 1A).

Effect of AZD4547 on clonogenicity of $4 T 1$ cells

To further determine whether AZD4547 could inhibit the proliferation of 4T1, we conducted clonogenic assay after AZD4547 treatment. As shown in Fig. 1B, AZD4547 suppressed colony formation of $4 \mathrm{~T} 1$ in a concentration-dependent manner. Furthermore, 
Table 1. The proliferation inhibitory effect of AZD4547 against cancer cell lines

\begin{tabular}{lll}
\hline Cell line & Cell type & $\mathrm{IC}_{50}(\mu \mathrm{M})$ \\
\hline 4T1 & mouse breast tumor cell line & $0.64 \pm 0.11$ \\
MDA-MB-468 & Human breast tumor cell line & $4.9 \pm 0.85$ \\
HCT116 & Human colorectal carcinoma cell line & $15.9 \pm 1.82$ \\
SW620 & Human colorectal carcinoma cell line & $>20$ \\
MDA-MB-231 & Human breast tumor cell line & $>20$ \\
CT26 & mouse colorectal carcinoma cell line & $>20$ \\
SW480 & Human colorectal carcinoma cell line & $>20$ \\
\hline
\end{tabular}

A

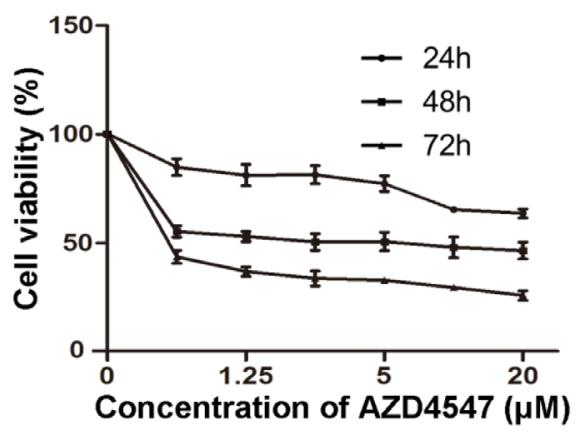

C

Control

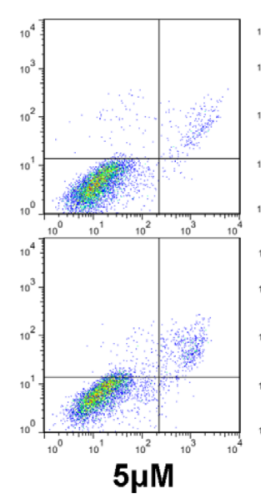

$1.25 \mu \mathrm{M}$

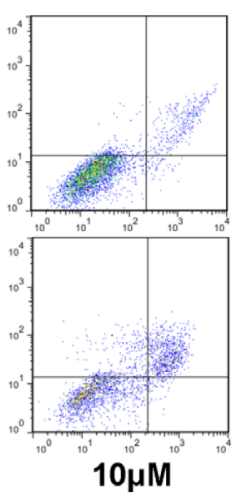

$2.5 \mu \mathrm{M}$
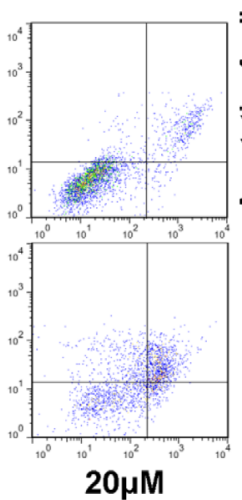

B
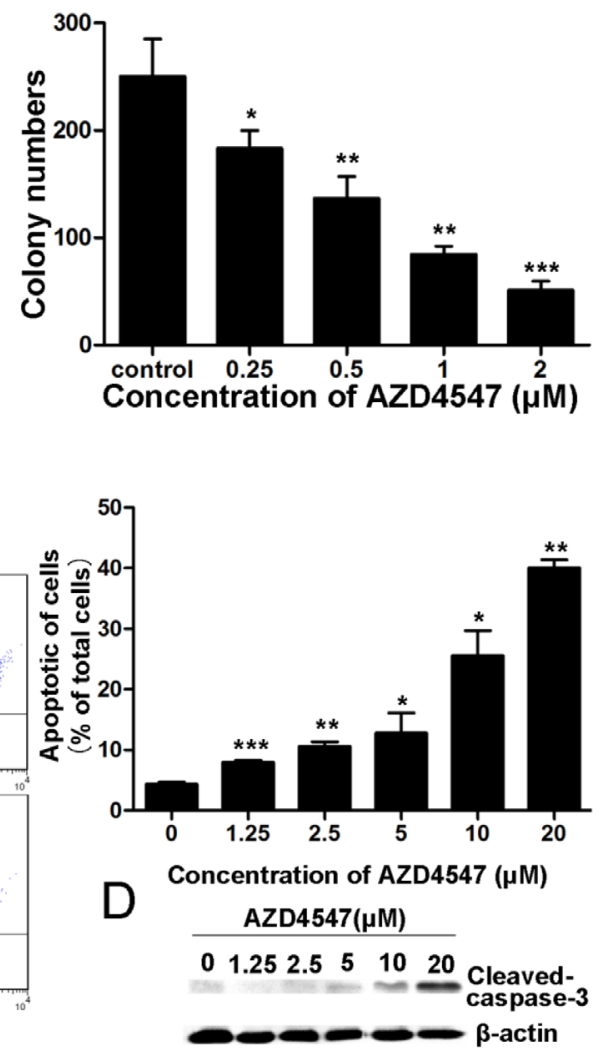

Fig. 1. The effect of AZD4547 on 4T1 cells viability and apoptosis progression. A MTT assay showed AZD4547 inhibited 4T1 cells proliferation concentration- and time-dependently. B The effects of AZD4547 $(0 \sim 2 \mu \mathrm{M})$ on colony formation in 4T1 cells for 10 days. Statistic results of colony-forming assays presented as surviving colonies. C Flow cytometric analysis of cells stained with Annexin V-FITC/PI after treatment AZD4547 $(0 \sim 20 \mu \mathrm{M})$ at $24 \mathrm{~h}$. Statistical results of apoptosis assays presented as apoptosising cells (percentage of untreated control). D The protein level of cleaved-caspase-3 was detected by Wwestern blot in 4T1 cells treated with AZD 4547 for $48 \mathrm{~h}$. Bars show mean \pm SD for at least 3 independent experiments. Statistical significance was assessed by unpaired t test. ${ }^{*} \mathrm{p}<0.05,{ }^{* *} \mathrm{p}<0.01,{ }^{* * *} \mathrm{p}<0.001$.

the size of the colonies treated with AZD4547 at the concentration of $2 \mu \mathrm{M}$ was significantly smaller than the control. The results from clonogenic assay were consistent with the MTT assay. Taken together, these results showed that AZD4547 can inhibit the proliferation of 4T1 cells. 


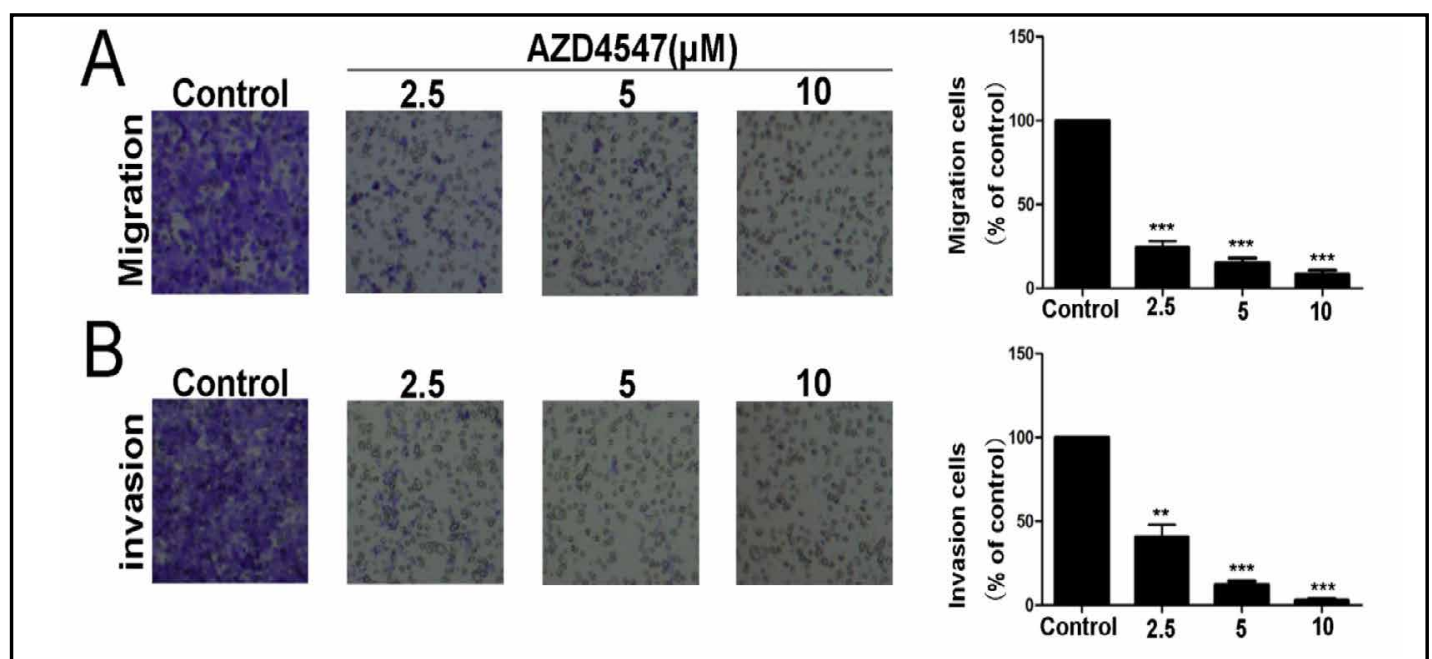

Fig. 2. The effect of AZD4547 on 4T1 cells migration and invasion. 4T1 cells migration (A) and invasion (B) were inhibited by AZD4547 in a dose dependent manner. Bars show mean \pm SD for at least 3 independent experiments. Statistical significance was assessed by unpaired t test. ${ }^{*} \mathrm{p}<0.05,{ }^{* *} \mathrm{p}<0.01,{ }^{* * *} \mathrm{p}<0.001$.

\section{AZD4547 induces cell apoptosis}

Previous studies have showed that AZD4547 significantly induced apoptotic in breast cell lines [24]. To elucidate the mechanism of AZD4547 to induce apoptosis in breast cancer in vitro, Annexin V-FITC and PI fluorescence staining was analyzed by FCM to further detect apoptosis. As Fig. 1C showed, the percentage of apoptosis cells increased from 8.3 $\%$ at $1.25 \mu \mathrm{M}$, to $13.5 \%$ at $5 \mu \mathrm{M}$, to $38.7 \%$ at $20 \mu \mathrm{M}$ after 24 hours of drug exposure. The results indicated that AZD4547 induced apoptosis of 4T1 cells in a concentration dependent manner after 24 hours treatment. To further confirm whether AZD4547-induced apoptosis was associated with activation of caspase family proteins, we examined the expression of cleaved-caspase-3 in 4T1 cells after AZD4547 treatment for 48 hours by Western blot. As depicted in Fig. 1D, Western blot analysis revealed that the production of cleaved-caspase-3 was significantly increased in a concentration-dependent manner. These results suggested that the AZD4547 induced suppression of 4T1 tumor cells is mediated by induction of the apoptosis through increasing the matured caspase-3.

\section{AZD4547 inhibits 4 T1 cells migration and invasion in vitro}

Fibroblast growth factors (FGFs), in a family of 23 different members, along with four tyrosine kinase FGF receptors, have been implicated in diverse cellular processes including cell apoptosis, adhesion and migration [25-27]. Recent studies showed that AZD4547 is a small-molecule FGFRs tyrosine kinase inhibitor [24]. Furthermore, the 4T1 mammary carcinoma is a transplantable tumor cell line that is highly tumorigenic and invasive and can spontaneously metastasize from the primary tumor in the mammary gland to multiple distant sites including lung, blood, lymph nodes and liver [28]. Taken together, it is imperative to research the ability of AZD4547 to inhibit 4T1 cell migration and invasion. The cell motility through an $8 \mu \mathrm{m}$ pore size polycarbonate membrane was tested by migration assay. The cell motility and the ability of tumor cells to invade through a basement membrane model were evaluated by invasion assay [29]. Firstly, as shown in Fig. 2A, through the transwell assay to investigate the effects of AZD4547 on 4T1 cells migration, we found that AZD4547 strongly decreased the number of cells migrated from the top chambers to the bottom chambers, indicating the inhibition of AZD 4547 on the 4T1 cells migration in a concentration-dependent manner. Similarly, AZD4547 also displayed an inhibited invasion capability according to the decreased number of invasive 4T1 cells (Fig. 2B). As described below, we also examined the effects of AZD4547 on lung metastases in vivo. 
Fig. 3. The effect of AZD4547 on 4T1 tumor model in vivo. A $4 \mathrm{~T} 1$ cells were established subcutaneous in female BALB/c mice. AZD4547 at $5 \mathrm{mg} /$ $\mathrm{kg}$ and control administration were started 7 days after inoculation. Every other day by intraperitoneal injection treatment was continued for 20 days. The mean tumor volumes \pm SD of 6 mice per every group. B The mean mice weight \pm SD of every group. Control and treated photographs (C) and weight (E) of tumors were presented respectively. Normal (that is not bearing tumor), control and treated photographs (D) and weight (F) of spleens were presented respectively. Bars show mean \pm SD. Statistical significance was assessed by unpaired t test. ${ }^{*} \mathrm{p}<0.05,{ }^{* *} \mathrm{p}<0.01$, $* * * \mathrm{p}<0.001$.

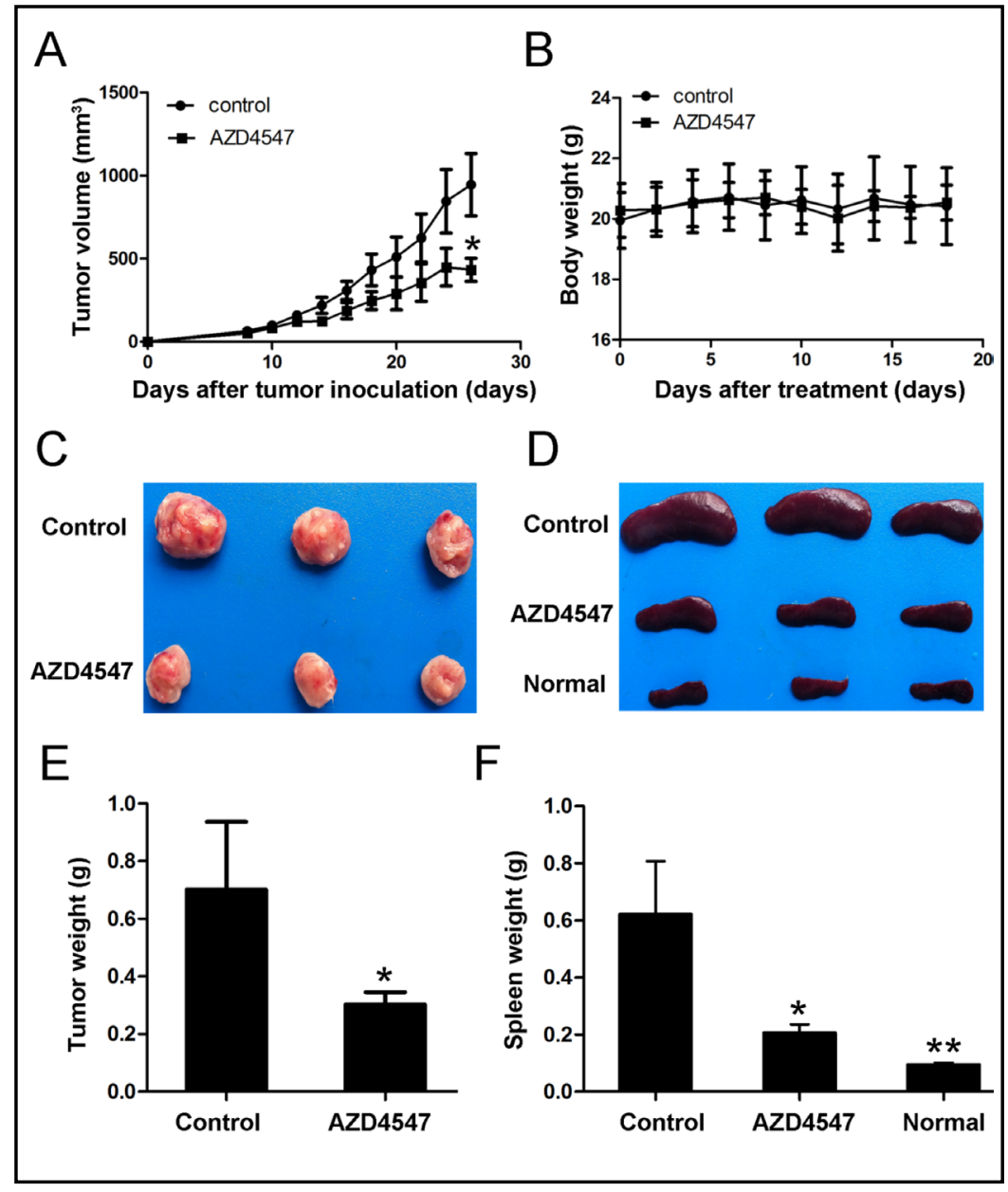

\section{Anti-tumor activity of AZD4547 in vivo}

To determine AZD4547 anti-tumor activity in vivo, 4T1 cells were injected subcutaneously into the right flanks of BALB/c mice to establish xenografts, as shown in Fig. 3A and Fig. 3C, AZD4547 remarkably decreased 4T1 tumor volume by about $60 \%$ compared with untreated groups. At the same time, AZD4547 treatment was well tolerated and did not cause significant loss in body weight (Fig. 3B). Importantly, no obvious changes were observed, including serum biochemistry and hematology parameters (date not shown). Furthermore, accumulating evidence since the 1980s has demonstrated that MDSCs are significantly increased in spleens of animals bearing tumors and in conditions associated with impaired immune reactivity [30]. As shown in Fig. 3D and Fig. 3F, AZD4547-treated groups didn't induce large splenomegaly compared with the untreated groups, and only slightly large splenomegaly compared with normal groups.

\section{AZD4547 blocks lung metastases}

It has been shown that 4T1 mammary tumor model shares many characteristics with human breast cancer, especially both have a high lung metastatic potential [31]. Previous studies have suggested that 4T1 tumor lethality is due to early metastasis, among which lung metastases could better represent the level of 4T1 cells spreading throughout the mouse $[31,32]$. Based on these considerations, when the tumor volume reached $1,000 \mathrm{~mm}^{3}$ in size, mice were sacrificed. Lung weight was measured, metastatic lung nodules were counted, and each metastatic focus more than $3 \mathrm{~mm}$ in diameter was measured under the dissecting microscope. As shown in Fig. 4A and Fig. 4C, there was two-fold decrease in lung weight 
A

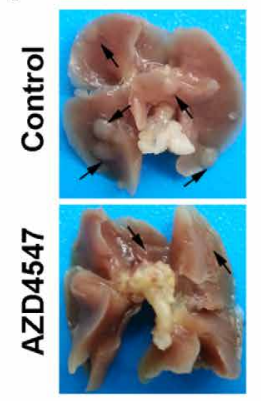

B

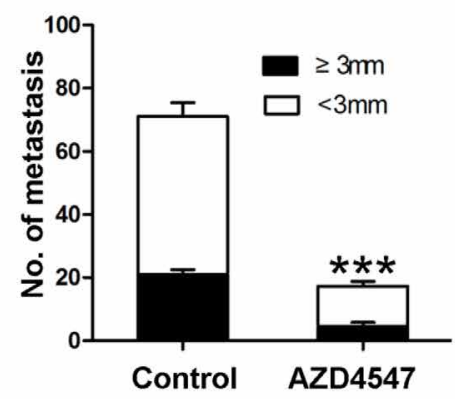

\section{C}

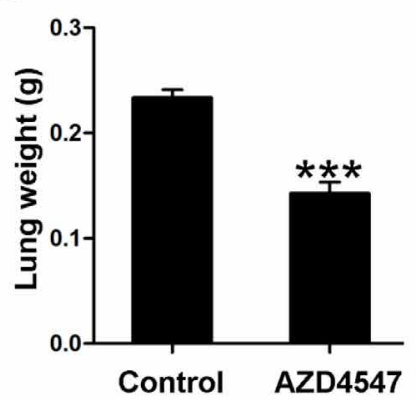

D
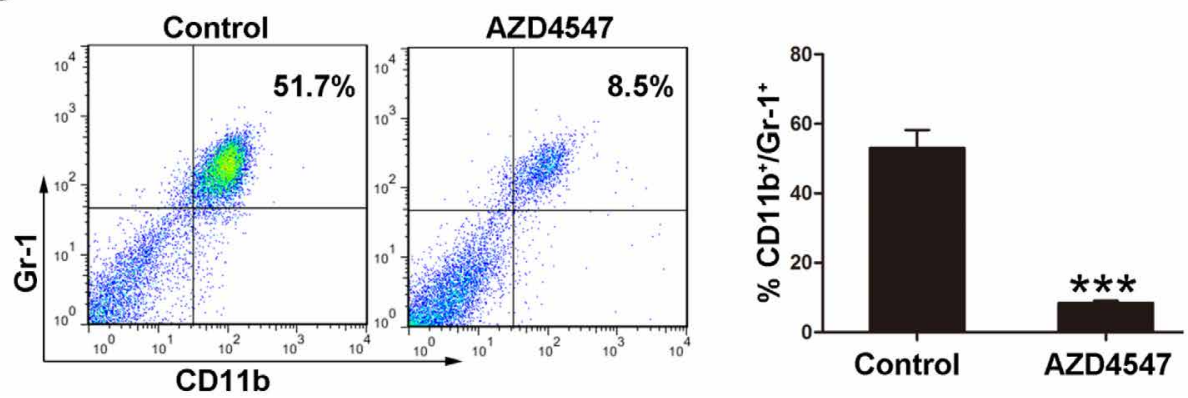

Fig. 4. AZD4547 inhibits spontaneous lung metastasis of 4T1 tumor-bearing mice. A The difference of lung nodules present in individual mice. B The mean lung nodules of every group. C The mean lung weight of every group. D FCM evaluating lung CD11 $\mathrm{b}^{+} / \mathrm{Gr} 1^{+}$myeloid cells isolated from 4T1 tumor-bearing mice after 20 days of treatment with AZD4547 or control. Statistical significance was assessed by unpaired t test. * $\mathrm{p}<$ $0.05,{ }^{* *} \mathrm{p}<0.01,{ }^{* * *} \mathrm{p}<0.001$.

by the in AZD4547 treatment group compared to the untreated control. More interestingly, the number of lung metastatic nodules was significantly reduced after 20 days of AZD4547 treatment compared with vehicle (Fig. 4B), which further supported the notion that AZD4547 treatment inhibited lung metastases.

It has been shown that MDSCs have been closely related with lung metastases in breast cancer $[4,27]$. We therefore investigated whether AZD4547 inhibited lung metastases through inhibiting tumor-induced MDSCs accumulation in lung. To confirm it, we design FCM assay. Single cell suspensions from lung were harvested and MDSCs with Gr-1+ $/$ CD11 $\mathrm{b}^{+}$ markers were analyzed. As shown in Fig. 4D, in untreated tumor-bearing mice, MDSCs made up to $53.1 \%$ of lung cells, whereas the AZD 4547 treatment reduced the proportion of $\mathrm{Gr}-1^{+}$/ CD11 $\mathrm{b}^{+}$cells to $8.4 \%$.

\section{AZD4547 mediated specific anti-tumor immunity.}

It has been reported that MDSCs were closely related to a metastatic tumor burden in patients with breast cancer and they are significantly infiltrating and accumulating in lung, tumor, spleens and peripheral blood of tumor-bearing mice $[8,9]$. Our data in the current study indicated that AZD4547 can inhibit lung metastases in mice bearing 4T1 tumors. We then investigated whether AZD4547 reduced MDSCs in tumor, peripheral blood and spleens was related to improving anti-tumor responses and cancer immunotherapy. Twenty days after the treatment, single cell suspensions from tumor, peripheral blood and spleens of 4T1 tumor-bearing mice were harvested to analyze the number of MDSCs. As shown in Fig. 5, MDSCs in the peripheral blood, spleens and tumors were obviously decreased after AZD4547 treatment, consistent with the reduction of MDSCs in the lung in AZD4547-treated groups (Fig. 4). These agree with previous results that AZD4547 treatment significantly reduced the weight of spleens as well as the nodule of lung metastatic compared with untreated groups. 


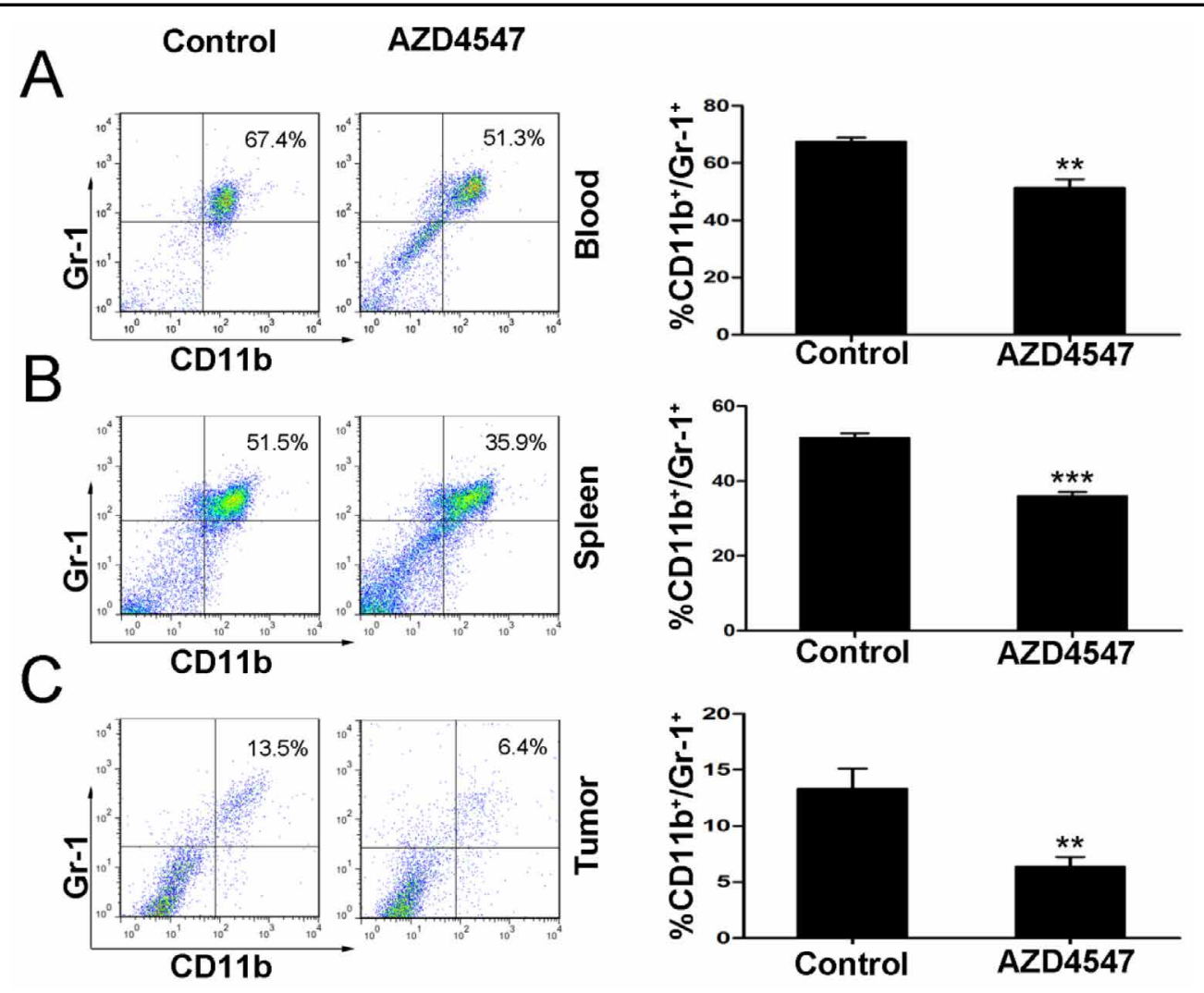

Fig. 5. Effects of MDSCs inhibition by AZD4547 on the tumor microenvironment. Treatment with AZD4547 reduces peripheral blood (A), spleens (B) and tumor (C) MDSCs infiltration. FCM evaluating peripheral blood, spleens and tumor CD11 $\mathrm{b}^{+} / \mathrm{Gr} 1^{+}$MDSCs isolated from 4T1 tumor-bearing mice after 20 days of treatment with AZD4547 or control. Bars show mean \pm SD ( 3 independent experiments with 3 mice each group). Statistical significance was assessed by unpaired t test. ${ }^{*} \mathrm{p}<0.05,{ }^{* *} \mathrm{p}<0.01,{ }^{* * *} \mathrm{p}<0.001$.

\section{AZD4547 treatment induced the production of $C D 4^{+}$and $C D 8^{+}$T-cells}

To further elucidate the mechanism of AZD4547 to mediate specific anti-tumor immunity, we focus on the role of T-cells. In the study, single cell suspensions from the tumor and spleens tissues were harvested to analyze the change of proportions of CD4 ${ }^{+}$and $\mathrm{CD} 8^{+}$ T-cells after AZD4547 treatment. As shown in Fig. 6, the frequencies of CD4 ${ }^{+}$and CD8 ${ }^{+}$T-cells in the tumor sections were increased from $2.52 \%$ and $1.35 \%$ to $6.63 \%$ and $2.05 \%$ after AZD4547 treatment, respectively. In addition, the proportion of CD4 ${ }^{+}$and $\mathrm{CD}^{+} \mathrm{T}$-cells in the spleen was also significantly increased to $13.34 \%$ and $3.36 \%$ from $6.91 \%$ and $1.59 \%$ after the treatment respectively. These data suggested that AZD4547 could also mediate specific anti-tumor immunity and efficiency through promoting the production and accumulation of $\mathrm{CD} 4^{+}$and $\mathrm{CD} 8^{+} \mathrm{T}$-cells in vivo.

\section{Discussion}

AZD4547 is a selective small-molecule inhibitor against the tyrosine kinase of FGFRs. In light of previous reports, it could significantly inhibit FGFR phosphorylation and repress proliferation of non-small cell lung cancer, breast cancer and gastric cancer cell lines via inhibition of FGFR signaling and it is now under phase II clinical study in human subject breast cancer $[17,18]$. However, there have been few reports regarding the corresponding molecule mechanism of AZD4547 against breast cancer. Importantly, it is unknown as to 


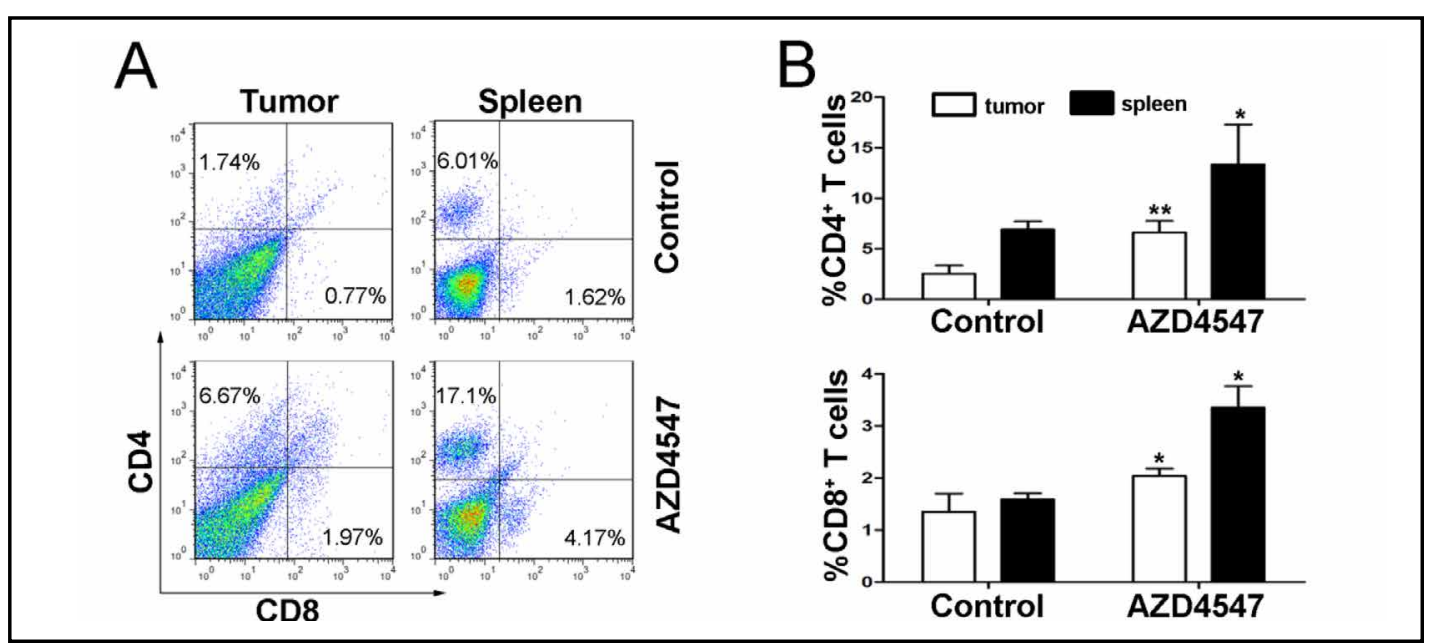

Fig. 6. AZD4547 treatment of $4 \mathrm{~T} 1$ tumor-bearing mice upregulates $\mathrm{CD} 4^{+}$and $\mathrm{CD}^{+}{ }^{+} \mathrm{T}$-cells. Treatment with AZD4547 upregulates spleens and tumor T-cells. FCM evaluating spleens and tumor CD4 ${ }^{+} / \mathrm{CD}^{+} \mathrm{T}$ cells isolated from 4T1 tumor-bearing mice after 20 days of treatment with AZD4547 or control. Bars show mean \pm $\mathrm{SD}$ (3 independent experiments with 3 mice each group). Statistical significance was assessed by unpaired $t$ test. ${ }^{*} \mathrm{p}<0.05,{ }^{* *} \mathrm{p}<0.01,{ }^{* * *} \mathrm{p}<0.001$.

whether the tumor suppression activities of AZD4547 are mediated by modulating immune homeostasis in addition to direct killing of the tumor cells. In the present study, we found AZD4547 not only can directly suppress the proliferation of breast cancer cell lines, but also restrain MDSCs and therefore restore immune surveillance, leading clearance of 4T1 tumor in the immune competent mice. The latter finding, although the underlying mechanism remains unknown, may open a new avenue by targeting the tumor exerted immune tolerance, and therefore restoring tumor immune surveillance. It is also possible that FGFR may abundantly present in the MDSCs, a subject under our investigation. On the other hand, additional work should be carried out to address how MDSCs suppress tumor-specific CD4 $4^{+}$and $\mathrm{CD} 8^{+} \mathrm{T}$ cells in the tumor environment and how $\mathrm{CD}^{+}{ }^{+}$and $\mathrm{CD}^{+} \mathrm{T}$ cells suppress $4 \mathrm{~T} 1$ cells in vitro. We will research the relationship between the $\mathrm{CD} 4^{+} / \mathrm{CD} 8^{+} \mathrm{T}$ cells and $4 \mathrm{~T} 1$ cells for their proliferation, apoptosis, migration, and invasion in the future.

Previous studies have identified FGFRs overexpression in breast cancer [33, 34]. In addition, it has been reported that inhibiting FGFRs signaling in the melanoma cells lead to tumor growth inhibition via massive induction of cell apoptosis [35]. Consistently, here we found that AZD4547 can induce apoptosis of 4T1 cells. It is unknown as to how AZD4547 promotes apoptosis of 4T1 cells. It is openly possible that AZD4547 may inhibit the activity of FGFRs, which is essential to restrain apoptosis and critical for the tumor cell survival.

Breast cancer comprises a heterogeneous group of malignancies derived from the ductal epithelium, which has a high metastatic potential, especially to lung $[5,26]$. The microenvironment of breast cancer is now recognized as a critical participant in tumor progression and metastasis $[5,36]$. Accumulating evidences since the 1980 s has demonstrated that number of MDSCs is strongly increased in the lung of breast tumor-bearing mice and in conditions they are associated with impaired immune reactivity. These cells are also found in the peripheral blood and spleens of lung, breast, head and neck cancer patients [30]. Non-immunological functions of MDSCs have also been described, such as the promotion of tumor-cell invasion and metastasis and tumor angiogenesis [8]. Transwell assay indicated that AZD4547 displayed inhibitory effects on 4T1 cells invasion and metastasis in vitro. In addition, we also investigated the anti-tumor effect of AZD4547 using 4T1 tumor model in vivo, which is a syngeneic mouse mammary carcinoma model that spontaneously develops lung metastases [4]. The results suggested that AZD4547 significantly inhibited tumor 
growth without causing obvious toxicities and the number of lung metastatic nodules was strongly reduced after AZD4547 treatment compared with vehicle treatment. Meanwhile, we examined lung-infiltrating MDSCs in 4T1 tumor-bearing mice by FCM and observed a reduction of lung-infiltrating MDSCs after AZD4547 treatment. The significant increase of MDSCs is consistent with increased lung metastases in untreated tumor-bearing mice, suggesting that these MDSCs may play a central role in breast tumor metastasis. These results confirmed that AZD4547 strongly inhibited the migration of 4T1 through inhibiting tumor-induced MDSCs accumulation.

MDSCs represent an intrinsic part of the myeloid-cell lineage that is comprised of myeloid-cell precursors and progenitors of myeloid cells [7]. Immunological function of MDSCs is to suppress T-cells proliferation. To such regard, we investigated whether enhanced number of T-cells was due to suppressing the infiltration and accumulation of MDSCs in the tumor, peripheral blood and spleens after AZD 4547 treatment. The current studies showed that not only number of MDSCs was decreased from $51.5 \%$ to $35.9 \%$ and $67.4 \%$ to 51.3 $\%$ in spleen cells and peripheral blood mononuclear cells of tumor-bearing mice but also proportion of $\mathrm{CD}^{+}$and $\mathrm{CD}^{+} \mathrm{T}$-cells in the tumor and spleens was increased after AZD4547 treatment, respectively, which displayed that AZD4547 strikingly reduced the expansion of MDSCs, resulting in increased $\mathrm{CD} 4^{+}$and $\mathrm{CD} 8^{+} \mathrm{T}$-cells responses.

In summary, our data indicated that AZD4547 was effective in inhibiting tumor growth and lung metastases without obvious side effect in the 4T1 tumor-bearing mice. To the best of our knowledge, the relevant mechanism study of AZD 4547 on breast cancer demonstrated that AZD4547 suppressed tumor metastasis and subsequent growth through inhibiting tumor-associated MDSCs accumulation to restore the CD4 ${ }^{+}$and $\mathrm{CD}^{+}{ }^{+} \mathrm{T}$-cells, makes AZD4547 an attractive therapeutic candidate for breast cancer.

\section{Abbreviations}

FGFRs (Fibroblast growth factor receptors); MDSCs (myeloid-derived suppressor cells); IMCs (immature myeloid cells); DMSO (Dimethyl Sulfoxide); MTT (3-(4, 5)-dimethylthiahiazo (-z-y1) -2, 5 -di-phenytetrazolium bromide); DMEM (Dulbecco's Modified Eagle's Medium); FBS (fetal bovine serum); PBS (phosphate-buffered saline); FCM (Flow Cytometry); PI (propidium iodide); SDS-PAGE (sodium dodecyl sulfate-polyacrylamide gel electrophoresis); PVDF (polyvinylidene fluoride); FGFs (Fibroblast growth factors).

\section{Conflict of Interest}

None declared.

\section{Acknowledgements}

This work was funded by the National Natural Science Foundation of China (81123003).

\section{References}

1 Siegel R, Naishadham D, Jemal A: Cancer statistics, 2013. CA: Cancer J Clin 2013;63:11-30.

2 Wang W, Eddy R, Condeelis J: The cofilin pathway in breast cancer invasion and metastasis. Nat Rev Cancer 2007; 7:429-440.

- Z Zhang T, Li J, Dong Y, Zhai D, Lai L, Dai F, Deng H, Chen Y, Liu M, Yi Z: Cucurbitacin E inhibits breast tumor metastasis by suppressing cell migration and invasion. Breast Cancer Res Tr 2012;135:445-458. 
Liu et al.: AZD4547 Inhibits Metastasis in Breast Tumor Model

-4 Yan HH, Pickup M, Pang Y, Gorska AE, Li Z, Chytil A, Geng Y, Gray JW, Moses HL, Yang L: Gr-1+ CD11b+ myeloid cells tip the balance of immune protection to tumor promotion in the premetastatic lung. Cancer Res 2010;70:6139-6149.

5 Place AE, Jin Huh S, Polyak K: The microenvironment in breast cancer progression: biology and implications for treatment. Breast Cancer Res 2011;13:227-238.

6 Cheng P, Corzo CA, Luetteke N, Yu B, Nagaraj S, Bui MM, Ortiz M, Nacken W, Sorg C, Vogl T: Inhibition of dendritic cell differentiation and accumulation of myeloid-derived suppressor cells in cancer is regulated by S100A9 protein. J Exp Med 2008;205:2235-2249.

7 Ostrand-Rosenberg S, Sinha P: Myeloid-derived suppressor cells: linking inflammation and cancer. J Immunol 2009;182:4499-4506.

8 Gabrilovich DI, Nagaraj S: Myeloid-derived suppressor cells as regulators of the immune system. Nat Rev Immunol 2009;9:162-174.

-9 Kodumudi KN, Woan K, Gilvary DL, Sahakian E, Wei S, Djeu JY: A novel chemoimmunomodulating property of docetaxel: suppression of myeloid-derived suppressor cells in tumor bearers. Clin Cancer Res 2010;1618:4583-4594.

10 Dolcetti L, Marigo I, Mantelli B, Peranzoni E, Zanovello P, Bronte V: Myeloid-derived suppressor cell role in tumor-related inflammation. Cancer Lett 2008;267:216-225.

11 Dugast A-S, Haudebourg T, Coulon F, Heslan M, Haspot F, Poirier N, de Silly RV, Usal C, Smit H, Martinet B: Myeloid-derived suppressor cells accumulate in kidney allograft tolerance and specifically suppress effector T cell expansion. J Immunol 2008;180:7898-7906.

12 Ray ME, Yang ZQ, Albertson D, Kleer CG, Washburn JG, Macoska JA, Ethier SP: Genomic and expression analysis of the 8p11-12 amplicon in human breast cancer cell lines. Cancer Res 2004;64:40-47.

13 Keats JJ, Reiman T, Belch AR, Pilarski LM: Ten years and counting: so what do we know about t (4; 14)(p16; q32) multiple myeloma. Leukemia Lymphoma 2006;47:2289-2300.

14 Pollock P, Gartside M, Dejeza L, Powell M, Mallon M, Davies H, Mohammadi M, Futreal P, Stratton M, Trent J: Frequent activating FGFR2 mutations in endometrial carcinomas parallel germline mutations associated with craniosynostosis and skeletal dysplasia syndromes. Oncogene 2007;26:7158-7162.

15 Jang J-H, Shin K-H, Park J-G: Mutations in fibroblast growth factor receptor 2 and fibroblast growth factor receptor 3 genes associated with human gastric and colorectal cancers. Cancer Res 2001;61:3541-3543.

16 Sahadevan K, Darby S, Leung H, Mathers M, Robson C, Gnanapragasam V: Selective over-expression of fibroblast growth factor receptors 1 and 4 in clinical prostate cancer. J Pathol 2007;213:82-90.

17 Zhang J, Zhang L, Su X, Li M, Xie L, Malchers F, Fan S, Yin X, Xu Y, Liu K: Translating the Therapeutic Potential of AZD4547 in FGFR1-Amplified Non-Small Cell Lung Cancer through the Use of Patient-Derived Tumor Xenograft Models. Clin Cancer Res 2012;18:6658-6667.

18 Xie L, Su X, Zhang L, Yin X, Tang L, Zhang X, Xu Y, Gao Z, Liu K, Zhou M: FGFR2 gene amplification in gastric cancer predicts sensitivity to the selective FGFR inhibitor AZD4547. Clin Cancer Res 2013;19:2572-2583.

19 Chen X, Ji P, Yang H-W, Yang L-L, Zhou S, Zhong L, Ma S, Fu X-Y, Zhou C, Li G-B: SC-535, a Novel Oral Multikinase Inhibitor, Showed Potent Antitumor Activity in Human Melanoma Models. Cell Physiol Biochem 2013;32:138-153.

20 Yi ZF, Cho SG, Zhao H, Wu Yy, Luo J, Li D, Yi T, Xu X, Wu Z, Liu M: A novel peptide from human apolipoprotein (a) inhibits angiogenesis and tumor growth by targeting c-Src phosphorylation in VEGF-induced human umbilical endothelial cells. Int J Cancer 2009;124:843-852.

-21 Dai XY, Zeng XX, Peng F, Han YY, Lin HJ, Xu YZ, Zhou T, Xie G, Deng Y, Mao YQ Yu LY, Yang L, Zhao YL: A novel anticancer agent, sklb70359, inhibits human hepatic carcinoma cells proliferation via g0/g1 cell cycle arrest and apoptosis induction. Cell Physiol Biochem 2012;29:281-290.

22 Xuejiao S, Yong X, Ningyu W, Lidan Z, Xuanhong S, Youzhi X, Tinghong Y, Yaojie S, Yongxia Z, Luoting Y: A Novel Benzothiazole Derivative YLT322 Induces Apoptosis via the Mitochondrial Apoptosis Pathway In Vitro with Anti-Tumor Activity in Solid Malignancies. PloS One 2013;8:e63900.

23 Kortylewski M, Kujawski M, Wang T, Wei S, Zhang S, Pilon-Thomas S, Niu G, Kay H, Mulé J, Kerr WG: Inhibiting Stat3 signaling in the hematopoietic system elicits multicomponent antitumor immunity. Nat Med 2005;11:1314-1321.

24 Gavine PR, Mooney L, Kilgour E, Thomas AP, Al-Kadhimi K, Beck S, Rooney C, Coleman T, Baker D, Mellor MJ: AZD4547: an orally bioavailable, potent, and selective inhibitor of the fibroblast growth factor receptor tyrosine kinase family. Cancer Res 2012;72:2045-2056. 
25 Li J-S, Yao Z-X: Modulation of FGF receptor signaling as an intervention and potential therapy for myelin breakdown in Alzheimer's disease. Med Hypotheses 2013.

-26 Dey JH, Bianchi F, Voshol J, Bonenfant D, Oakeley EJ, Hynes NE: Targeting fibroblast growth factor receptors blocks PI3K/AKT signaling, induces apoptosis, and impairs mammary tumor outgrowth and metastasis. Cancer Res 2010;70:4151-4162.

27 Ye T, Wei X, Yin T, Xia Y, Li D, Shao B, Song X, He S, Luo M, Gao X: Inhibition of FGFR signaling by PD173074 improves antitumor immunity and impairs breast cancer metastasis. Breast Cancer Res Tr DOI: 10.1007/ s10549-013-2829-y.

28 Pulaski BA, Ostrand-Rosenberg S: Mouse 4T1 breast tumor model. Curr Protoc Immunol Doi: 10.1002/0471142735.im2002s39.

29 Tu Z, Ma Y, Tian J, Li H, Akers W, Achilefu S, Gu Y: Estrogen receptor $\beta$ potentiates the antiproliferative effect of raloxifene and affects the cell migration and invasion in HCT-116 colon cancer cells. J Cancer Res Clin Oncol 2012;138:1091-1103.

30 Yang L, DeBusk LM, Fukuda K, Fingleton B, Green-Jarvis B, Shyr Y, Matrisian LM, Carbone DP, Lin PC: Expansion of myeloid immune suppressor Gr+ CD11b+ cells in tumor-bearing host directly promotes tumor angiogenesis. Cancer Cell 2004;6:409-421.

-31 Zhang B, Ma X, Li Z, Gao X, Wang F, Liu L, Shen G, Sang Y, Li M, Li Y: Celecoxib enhances the efficacy of 15-hydroxyprostaglandin dehydrogenase gene therapy in treating murine breast cancer. J Cancer Res Clin Oncol 2013;1-11.

-32 Xin H, Herrmann A, Reckamp K, Zhang W, Pal S, Hedvat M, Zhang C, Liang W, Scuto A, Weng S: Antiangiogenic and antimetastatic activity of JAK inhibitor AZD1480. Cancer Res 2011;71:6601-6610.

-33 Liao D, Luo Y, Markowitz D, Xiang R, Reisfeld RA: Cancer associated fibroblasts promote tumor growth and metastasis by modulating the tumor immune microenvironment in a 4T1 murine breast cancer model. PLoS One 2009;4:e7965.

-34 Tiong KH, Mah LY, Leong C-O: Functional roles of fibroblast growth factor receptors (FGFRs) signaling in human cancers. Apoptosis 2013:1-22.

-35 Valesky M, Spang AJ, Fisher GW, Farkas DL, Becker D: Noninvasive dynamic fluorescence imaging of human melanomas reveals that targeted inhibition of bFGF or FGFR-1 in melanoma cells blocks tumor growth by apoptosis. Mol Med 2002;8:103-112.

-36 Park CC: The Stromal Microenvironment and Breast Cancer Biology. Breast Diseases: A Year Book Quarterly 2013;24:23-27. 